OU-TP-98-41P

QMW-PH-98-35

hep-th/9807223

July 1998

\title{
GRAVITATIONALLY DRESSED RG FLOWS AND ZIGZAG-INVARIANT STRINGS
}

\author{
IAN. I. Kogan 1, 网 AND O. A. Soloviev ${ }^{2}$, 四 \\ ${ }^{1}$ Theoretical Physics, Department of Physics, University of Oxford \\ 1 Keble Road, Oxford, OX1 3NP, United Kingdom \\ 2 Physics Department, Queen Mary and Westfield College, \\ Mile End Road, London E1 4NS, United Kingdom
}

\begin{abstract}
We propose a world-sheet realization of the zigzag-invariant bosonic and fermionic strings as a perturbed Wess-Zumino-Novikov-Witten model at large negative level $k$ on a group manifold $G$ coupled to $2 \mathrm{D}$ gravity. In the large $k$ limit the zigzag symmetry can be obtained as a result of a self-consistent solution of the gravitationally dressed RG equation. The only solution found for simple group is $G=S L(2)$. More general target-space geometries can be obtained via tensoring of various cosets based on $S L(2)$. In the supersymmetric case the zigzag symmetry fixes the maximal target-space dimension of the confining fermionic string to be seven.
\end{abstract}

*e-mail: i.kogan1@physics.ox.ac.uk

${ }^{\dagger}$ e-mail: O.A.Soloviev@QMW.AC.UK 


\section{Introduction}

A hunt for the string theory formulation of QCD has, recently, entered a very intriguing stage. A year ago Polyakov put forward a new idea about how to get a string description of a gauge field theory without running into a problem of having spin- 2 massless particles in the open string spectrum [1]. The main ingredient of this string formulation is a new symmetry on the world-sheet which he called zigzag symmetry. This is nothing but a symmetry under orientation changing world-sheet metric diffeomorphisms, $\operatorname{det}\left(\partial x^{\prime} / \partial x\right)<$ 0 . The power of the zigzag symmetry is that it singles out only vector states of the open string in the (D-dimensional) domain of the target-space to which Wilson loops of the confining string are attached. In other words, the zigzag symmetry gives rise to string models without gravity in the space-time.

A world-sheet description of the confining string appears to be fairly intricate [1], [2]. It looks to be a certain non-critical string whose world-sheet action is given by [1]

$$
S=\int d^{2} \xi\left[(\partial \phi)^{2}+a^{2}(\phi)(\partial x)^{2}+\Phi(\phi) R^{(2)} \sqrt{g}\right]
$$

where we omit possible antisymmetric fields, like $B_{\mu \nu}$ or Ramond-Ramond fields. Here $\phi$ is the Liouville field of $2 \mathrm{D}$ gravity, $x^{\mu}$ ( $\mu$ runs from 0 to $D-1$ ) are coordinates of the confining string, $\Phi$ is the dilaton field, $R^{(2)}$ is the curvature of the world-sheet and $a(\phi)$ is the running string tension. The zigzag symmetry requires the existence of a certain value of the Liouville field $\phi^{*}$ such that

$$
a\left(\phi^{*}\right)=0
$$

At the given point, the string representation of the Wilson loop is determined by the world-sheet action for antisymmetric $B_{\mu \nu}$ and/or R-R fields [1]. One can think about the surface of a zero string tension as a horizon in a curved $D+1$-dimensional space.

According to Polyakov's ansatz, the colour-electric flux of the D-dimensional gauge field theory propagates in at least $\mathrm{D}+1$ dimensions, so that the $\mathrm{D}$-dimensional gauge theory emerges on the boundary of a $(\mathrm{D}+1)$-dimensional space-time (with negative curvature). A very similar geometry of a target-space was recently used in a recent AdS/CFT construction for $N=4$ SUSY gauge theories [3], [4], [5]. 
The aim of the present paper is to take a step further towards a concrete worldsheet formulation of a string model which is invariant under the zigzag symmetry. To find relevant geometry one has to study the beta-function for $a(\phi)$. The major unsolved problem is to go beyond one-loop approximation for beta function which is invalid for asymptotically small $a(\phi)$ and large negative curvature near the horizon. In this paper we shall approach this problem by studying the gravitationally dressed RG flow [6], [7] describing the deformation of a non-compact WZNW model by the kinetic term operator in the large $k$ limit. We shall look for group manifolds such that a dressed RG flow has a nontrivial IR fixed point at which the kinetic term is canceled, i.e. at this point the target space metric is zero and we have pure Wess-Zumino term interacting with gravity. It is amusing that solution of this problem exists !

\section{Formulation of the bosonic zigzag-invariant string}

The main objective of the world-sheet formulation of the zigzag-invariant string is to obtain a conformal field theory which has a sector with the running coupling constant in front of the kinetic terms. Our first step will be to consider a certain non-conformal two-dimensional model with the running constant in front of the kinetic term. Such a theory has been discussed in [8]. It is a (non-unitary) Wess-Zumino-Novikov-Witten (WZNW) model perturbed by its kinetic term. In two-dimensional complex coordinates, the corresponding action is written as follows

$$
S_{\epsilon}=S_{W Z N W}(G, k)-\epsilon \int d^{2} z O(z, \bar{z})
$$

Here $\epsilon$ is a coupling constant,

$$
S_{W Z N W}(G, k)=\frac{k}{4 \pi} \int d^{2} z \operatorname{Tr}\left(g^{-1} \partial_{z} g \cdot g^{-1} \partial_{\bar{z}} g\right)+\Gamma(G, k)
$$

where the last term is a Wess-Zumino term

$$
\Gamma(G, k)=\frac{k}{12 \pi} \int_{M} d^{3} x \epsilon^{i j k} \operatorname{Tr}^{-1} \partial_{i} g \cdot g^{-1} \partial_{j} g \cdot g^{-1} \partial_{k} g,
$$

and

$$
O(z, \bar{z})=\frac{1}{c_{V}(G)} J^{a} \bar{J}^{b} \phi^{a b}
$$


where we use the following notations

$$
J \equiv J^{a} t^{a}=-\frac{k}{2} \partial g g^{-1}, \quad \bar{J} \equiv \bar{J}^{a} t^{a}=-\frac{k}{2} g^{-1} \bar{\partial} g,
$$

and also

$$
\phi^{a b}=\operatorname{Tr}\left(g^{-1} t^{a} g t^{b}\right), \quad c_{V}(G)=-\frac{f_{d}^{a c} f_{c}^{b d} \eta_{a b}}{\operatorname{dim} G} .
$$

The operator $O$ in eq. (2.6) has the following conformal dimension [9]

$$
\Delta_{0}=1+\frac{c_{V}(G)}{k+c_{V}(G)}
$$

which is positive and less than one if

$$
k<-2 c_{V}(G)
$$

This condition is important, since we want $O$ to be a relevant operator, so that the conformal point $\epsilon_{0}=0$ is unstable. The negative $k$ means that we are dealing with a non-unitary WZNW model: there will be states with negative norms in the spectrum. It is important that the operator $O$ itself generates a unitary highest weight representation of the Virasoro algebra, because we choose $\Delta_{0}$ be positive. Later we shall show that only the unitary subsector of the given theory will play a role.

Another important property of the operator $O$ is that it obeys the following fusion rule 10

$$
O \cdot O=[\mathbf{1}]+[O]+\ldots
$$

where the square brackets denote the contributions of $O$ and the identity operator $\mathbf{1}$ and their descendants, whereas dots stand for operators with conformal dimensions greater than one. The given OPE implies that the perturbed CFT is renormalizable.

Since the critical point $\epsilon_{0}=0$ is unstable, the theory (2.1) will flow towards some IR fixed point or to a massive phase. The corresponding renormalization group beta function of the coupling constant $\epsilon$ is given by [1], [12]

$$
\beta \equiv \frac{\mathrm{d} \epsilon}{\mathrm{d} \ln \Lambda}=\left(2-2 \Delta_{0}\right) \epsilon-\pi C \epsilon^{2}+\ldots
$$

where $\Lambda$ is the ultraviolet cutoff. Here the (three-point function) coefficient $C$ is normalized to 1 , due to the factor $1 / c_{V}(G)$ in the definition of $O$, eq.(2.4). Away from the 
conformal point $\epsilon_{0}$, the perturbed WZNW model is no longer conformal. However, it is easy to see that in the limit $k \rightarrow-\infty$, eq.(2.10) has a non-trivial fixed point

$$
\epsilon^{*}=-\frac{2 c_{V}(G)}{\pi k}
$$

at which the perturbed non-unitary WZNW model becomes a unitary WZNW model with level $|k|-2 c_{V}(G)[8]$. This can be checked by computing anomalous conformal dimensions and the Virasoro central charge.

However, the flow from $\epsilon_{0}$ to $\epsilon^{*}$ must be very peculiar. Indeed, in the middle of the flow, the parameter $\epsilon$ has to pass another point

$$
\epsilon_{W Z}=-\frac{c_{V}(G)}{\pi k}
$$

at which the deformation kills the sigma-model kinetic term and the resulting theory has only the Wess-Zumino term, i.e.

$$
S\left(\epsilon_{W Z}\right) \sim \Gamma
$$

This point does not appear to be special from the point of view of the beta function. For example, it is not a critical point. However, one can expect some very dramatic events happening as $\epsilon(t)$ approaches $\epsilon_{W Z}$, since the theory becomes purely topological. The properties of this theory are quite puzzling [13] which make fairly difficult its quantum interpretation. In spite of certain pathology, the described perturbed WZNW model seems to mimic well the behaviour of Polyakov's confining string. Indeed, we can identify the coordinates $x^{\mu}$ from ansatz (1.1) with the coordinates on the group manifold $G$. Then the variable tension will be given as follows

$$
a(t) \sim k^{2}\left(\frac{1}{4 \pi k}+\frac{\epsilon(t)}{4 c_{V}(G)}\right) .
$$

In order to make the link between the perturbed WZNW model and Polyakov's ansatz more precise, we have to couple the former to 2D gravity. We shall show that this coupling will fix the pathologies of the original theory (2.1).

The point to be made is that the Virasoro central charge of the unperturbed WZNW model is given as follows 9

$$
c_{W Z N W}(G, k)=\frac{k \operatorname{dim} G}{k+c_{V}(G)}
$$


and it is not necessarily equal to 26. Therefore, the given WZNW model has to be thought of as being a certain non-critical string. It is well-known that a non-critical string in $D$ dimensions with coordinates $x^{\mu}$ can be viewed as a critical string in the $(D+1)$ - dimensional space formed by $X^{M}=\left(x^{\mu}, \phi\right)$, with $\phi$ being the Liouville field.

As a result of the gravitational Ward identities, the interaction with 2D gravity makes any two-dimensional quantum field theory conformal. In particular, the perturbed WZNW model in question coupled to 2D gravity has to be a CFT. In other words, the gravitational interaction modifies the renormalization group properties of the system. The effects of the gravitational dressing have been studied in light-cone [14], [15] and conformal gauges in [16] [17], [7] and [6] (see also [18], [19], [20], [21] and references therein). In a light-cone gauge the renormalization of a one-loop beta function, i.e. constant $C$ was found for marginal operators [7]

$$
\bar{C}=\frac{\kappa+2}{\kappa+1} C=-\frac{2}{Q \alpha} C
$$

and in conformal gauge the result was obtained also for quasimarginal operators with bare dimensions $\Delta_{0}$ close to 1

$$
\bar{C}=-\frac{2}{\alpha\left(Q-2 \alpha_{1}\right)} C
$$

Let us note that it is still an open problem to obtain the result for $\Delta_{0} \neq 1$ in a light-cone gauge. For $c>25$ we have

$$
\begin{aligned}
& \kappa+2=\frac{1}{12}(c-13+\sqrt{(c-1)(c-25)}), Q=\sqrt{\frac{|c-25|}{3}}, \\
& \alpha=-\frac{Q}{2}+\frac{1}{2} \sqrt{Q^{2}+8}, \quad \alpha_{1}=-\frac{Q}{2}+\sqrt{\frac{Q^{2}}{4}-\left(2 \Delta_{0}-2\right)},
\end{aligned}
$$

The gravitationally dressed beta function is given by

$$
\bar{\beta} \equiv \frac{d \epsilon}{d \ln \Lambda}=(2-2 \Delta) \epsilon-\pi \bar{C} \epsilon^{2}+\ldots
$$

where $\Delta$ is the KPZ gravitational scaling dimension [15]

$$
\Delta-\Delta_{0}=\frac{\Delta(\Delta-1)}{\kappa+2}, \quad 1-\Delta=\frac{\alpha_{1}}{\alpha}
$$

One can see that there is a non-trivial IR fixed point

$$
\epsilon^{*}=-\frac{\alpha_{1}\left(Q-2 \alpha_{1}\right)}{\pi}
$$


One can trust this perturbative result only if $\left|\epsilon^{*}\right|<<1$ which is true in a large $k$ limit as will be shown later.

In order to find the Liouville field $\phi$ dependence of the gravitationally dressed $\beta$ function, we need a precise definition of the physical scale. In [6], [7] it was suggested to identify the scale $\Lambda^{-2}$ with the cosmological constant operator, also known as the "tachyon background" $T(\phi)$. Only its leading asymptotic for large $\phi$ is known $T(\phi) \sim e^{-\alpha \phi}$ and in this paper we are not going to address the issue of the intermediate $\phi$ behaviour. It is quite possible that $T(\phi)$ becomes zero at some finite $\phi$ and this will be the IR endpoint of the RG flow.

The well-known problem with the non-critical strings is that we do not know how to quantize them when the Virasoro central charge lies between 1 and 25. Therefore, in order to move forward with the theory in question, we have to make sure that its Virasoro central charge $\geq 25$. Otherwise, it might be difficult to construct any more or less realistic model of the confining string. As we shall see, our choice will be fully justified.

We can also assume that the zigzag-invariant string is confined to a low-dimensional domain of a larger space, that is there are extra coordinates $y^{n}$ which the zigzag-invariant string does not see. In other words, we assume that the Virasoro central charge without gravity has the following structure $c=c_{W Z N W}+c_{y}$ where $c_{y}$ is the Virasoro central charge associated with the additional coordinates $y^{n}$. The perturbation will be carried out only in the $x$-sector, leaving $y^{n}$ untouched.

In the limit $k \rightarrow-\infty$, the WZNW-Virasoro central charge has the following expansion in $1 / k$

$$
c_{W Z N W}(G, k)=\operatorname{dim} G+\frac{c_{V}(G) \operatorname{dim} G}{|k|}+\mathcal{O}\left(1 / k^{2}\right) .
$$

Thus, if we choose $\operatorname{dim} G$ and $c_{y}$ such that $\operatorname{dim} G+c_{y}=25$ then we shall have a noncritical string whose Virasoro central charge is just slightly greater than 25 . This condition will allow us to study the gravitational dressing using the $1 / k$-expansion method.

Within the described setup, we obtain the following asymptotic expressions:

$$
Q=\sqrt{\frac{c_{V}(G) \operatorname{dim} G}{3|k|}}+\ldots
$$




$$
\alpha_{1}=\sqrt{\frac{c_{V}(G)}{|k|}}\left[\frac{-\sqrt{\frac{\operatorname{dim} G}{3}}+\sqrt{\frac{\operatorname{dim} G}{3}+8}}{2}\right]+\ldots,
$$

where dots stand for higher in $1 / k$-corrections.

After substitution of the above formulas into eq.(2.21), we derive the following solution for the non-trivial fixed point in the presence of gravity

$$
\epsilon^{*}=-\frac{c_{V}(G)}{\pi|k|}\left(\frac{-\sqrt{\frac{\operatorname{dim} G}{3}}+\sqrt{\frac{\operatorname{dim} G}{3}+8}}{2}\right)\left(2 \sqrt{\frac{\operatorname{dim} G}{3}}-\sqrt{\frac{\operatorname{dim} G}{3}+8}\right) .
$$

One can see that the location of this critical point depends on the group $G$. In order for our theory to make a contact with Polyakov's confining string, we have to impose the following condition

$$
\epsilon^{*}=\epsilon_{W Z}=\frac{c_{V}(G)}{\pi|k|}
$$

which guarantees that the perturbed CFT coupled to 2D gravity runs to the zigzaginvariant background and settles down there. Eq.(2.25) results in an equation for $\operatorname{dim} G$

$$
\left(\sqrt{x^{2}+8}-2 x\right)\left(\sqrt{x^{2}+8}-x\right)=2, \quad x^{2}=\frac{\operatorname{dim} G}{3}
$$

and nobody promised us that the solution will give us a positive integer $\operatorname{dim} G$. Amusingly enough the solution is

$$
x^{2}=1, \quad \operatorname{dim} G=3 .
$$

Since the group $G$ has to be noncompact (because $k$ is negative), we end up with a unique solution

$$
G=S L(2)
$$

Correspondingly, we find that $c_{y}=22$. Because, the perturbation did not affect the $y$ sector, the latter stays completely factorized from both $x^{\nu}$ and the Liouville field throughout the flow.

In the limit $k \rightarrow-\infty$, our non-critical string with $G=S L(2)$ and $c_{y}=22$ at the UV-critical point will flow to the fixed point at which the $x$-sector of the theory acquires the zigzag symmetry. It seems to be appropriate to call eq.(2.25) the zigzag-anomaly free condition. The fact, that the zigzag symmetry fixes the group $G$ is not completely strange. We could have expected that the zigzag symmetry should have an anomaly at the 
quantum level (if we have started with a zigzag invariant classical model). In string theory, the anomaly cancellation always results in certain restrictions on the string background.

Condition (2.28) does not necessarily mean that the bosonic zigzag-invariant string can exist only in the three-dimensional space formed by the renormalization flow of the WZNW model on $S L(2)$ coupled to $2 \mathrm{D}$ gravity. The zigzag symmetry also can be realized via deformations of the $S L(2) / U(1)$ coset [22] which will lead to a certain two- dimensional zigzag-invariant string (see appendix). The $U(1)$-gauge invariant perturbation operator $\tilde{O}$ is defined as follows 22

$$
\tilde{O}=\frac{1}{c_{V}(G)}\left[J^{a} \bar{J}^{b} \phi^{a b}+\left(1+\frac{4}{k}\right) J^{a} \bar{J} \phi^{a 3}+\left(1+\frac{4}{k}\right) J \bar{J}^{b} \phi^{3 b}+\left(1+\frac{4}{k}\right)^{2} J \bar{J} \phi^{33}\right]
$$

where $J$ is the affine current associated with the subgroup $U(1)$.

The gateway to higher dimension formulations of the zigzag-invariant string opens up via tensoring of the WZNW model on the non-compact $S L(2)$ al level $k$ with the WZNW model on the compact $S U(2)$ at level $l$. It turns out that the level-dependence in the sum of the Virasoro central charges of these two WZNW models can vanish. Indeed, the sum is given as follows

$$
c_{\text {sum }}=\frac{3 k}{k+2}+\frac{3 l}{l+2}=6-6\left(\frac{1}{\tilde{k}}+\frac{1}{\tilde{l}}\right)
$$

where we made the following change of the levels $k=\tilde{k}-2, l=\tilde{l}-2$. So if we take $\tilde{l}=-\tilde{k}$ then $c_{\text {sum }}=6$ and does not depend on the levels. Obviously, $k$ has to be negative integer in order for the WZNW model on $S U(2)$ to be well defined.

Thus, via tensoring of one WZNW model on $S L(2)$ at level $k$ with the two WZNW models described above, we will get a system whose $Q$ has exactly the same $k$-dependence as for a single WZNW model on $S L(2)$. This is sufficient for the deformed two WZNW models on the non- compact groups to flow to the zigzag-invariant fixed point. In the case of two WZNW models on $S L(2)$, we obtain a six-dimensional zigzag-invariant string. The compact sector (formed by $y^{m}$ ) will have one $S U(2)$ and an additional 16-dimensional compact space, so that the whole target space, including the Liouville mode, will be 26-dimensional. We can also consider tensoring cosets. This way we can derive zigzaginvariant strings in dimensions from $D=2$ to $D=23$, though the total number of the 
target space dimensions will be always 26 . The dimension $D=23$ is maximal, since the compensating WZNW model (or its coset) on $S U(2)$ will have dimension, at least, equal to 2 , which will be the minimal dimension of the compact space.

\section{The zigzag-invariant string with the world-sheet supersymmetry}

The philosophy of the world-sheet formulation of the supersymmetric zigzag-invariant string almost completely repeats the bosonic case. The differences appear only in numerical details which, however, will be shown to lead us to quite remarkable consequences compared to the purely bosonic formulation. We restrict our consideration to the case of $N=1$ supersymmetry.

It is convenient to use the $N=1$ superspace formulation of the WZNW model [23]. The $N=1$ supergravity dressing has been studied in 24, 25, 26] and here we shall skip details which could be found in this publications. The gravitationally dressed beta function is given by the expression similar to (2.19)

$$
\hat{\beta} \equiv \frac{d \epsilon}{d \ln \Lambda}=2 \frac{\hat{\alpha}_{1}}{\hat{\alpha}} \epsilon+\frac{2 \pi}{\hat{Q}-2 \hat{\alpha}_{1}} \epsilon^{2}+\ldots
$$

and the IR fixed point is $\epsilon^{*}=-\hat{\alpha}_{1}\left(\hat{Q}-2 \hat{\alpha}_{1}\right) / \pi$. Here $\hat{\alpha}_{1}$ and $\hat{Q}$ are supersymmetric counterparts of the bosonic $\alpha_{1}$ and $Q$. They are given as follows

$$
\hat{Q}=\sqrt{\frac{\hat{c}-9}{2}} \quad \hat{\alpha}_{1}=-\frac{\hat{Q}}{2}-\sqrt{\frac{\hat{Q}^{2}}{4}-2\left(\hat{\Delta}_{0}-\frac{1}{2}\right)}
$$

where $\hat{c}$ is the $N=1$ super-Virasoro central charge and bare anomalous dimension of a deformation operator is

$$
\hat{\Delta}_{0}=\frac{1}{2}+\frac{\left.c_{V}(G)\right)}{k}
$$

It is related to the Virasoro central charge $c$ of the component theory as

$$
\hat{c}=\frac{2}{3} c
$$

The coefficient $2 / 3$ will turn out to be absolutely crucial. 
We would like to consider a supersymmetrical CFT whose super-Virasoro central charge $\hat{c}$ is slightly greater than 9 . This may require extra dimensions in the target space in addition to the supersymmetrical WZNW model on $G$. Thus, as in the bosonic case, the target space will consist of the $x$-sector and the $y$-sector, where $x^{\mu}$ and $y^{n}$ are now $N=1$ superfields. The dimensions of the given sectors are related as follows $\operatorname{dim} G+\hat{c}_{y}=9$. In the limit $k \rightarrow-\infty$, we find

$$
\hat{c}-9=\frac{2}{3} c-9=\frac{2 c_{V}(G) \operatorname{dim} G}{3|k|}
$$

which is, in fact, an exact expression (without higher in $1 / k$-terms). Substitution of the last expression and $\hat{\Delta}_{0}$ into (3.2) gives rise to exactly the same expressions for $\hat{\alpha}_{1}$ and $\hat{Q}$ as in bosonic eqs. 2.24) due to the factor $2 / 3$ in formula (3.4), which means the same value for $\epsilon^{*}$. Therefore, in the $N=1$ supersymmetric case, we shall arrive at the same zigzag anomaly cancellation condition as in (2.27), that is the group $G$ still has to be $S L(2)$. This allows us to construct a three-dimensional zigzag-invariant string with $N=1$ supersymmetry on the world-sheet. It is also straightforward to obtain a two-dimensional $N=1$ zigzag- invariant string based on the super-coset $S L(2) / U(1)$.

The advantage of the supersymmetric case compared to the bosonic one is that the $N=$ 1 supersymmetry lowers the number of possible $N=1$ supersymmetrical zigzag-invariant strings in higher dimensions. Indeed, let us consider two $N=1$ supersymmetric WZNW models defined on $S L(2)$ at level $k$ and $S U(2)$ at level $l$, correspondingly. Remarkably, the sum of their super-Virasoro central charges does not depend on the Kac-Moody level, if $l=-k$

$$
\hat{c}(S L(2), k)+\hat{c}(S U(2),-k)=\frac{2}{3}\left[\frac{3(k-2)}{(k-2)+2}+\frac{3(-k-2)}{(-k-2)+2}+3\right]=6
$$

Because of this cancellation, we can tensor these two WZNW models (or their gauged versions) with one $N=1$ supersymmetric WZNW model on $S L(2)$ (or the corresponding coset) at the large negative level $k$. Since $k$ is related to the Kac-Moody level of the WZNW model on a compact group, it has to be negative integer. However, this condition does not affect our computations. This level-cancellation mechanism is similar to the procedure considered in the bosonic case. However, in the supersymmetric case, the Kac-Moody levels come up already shifted by the right number. 
Let us describe how one can obtain a $D=4$ zigzag-invariant string. In order to get a four-dimensional space formed by $x^{\mu}$, we have to take two super-cosets $\frac{S L(2)}{U(1)}$ at the same level $k$. Each of the given cosets is deformed by the corresponding gauge invariant operator $\hat{\tilde{O}}$. The gauge invariant supersymmetric operator $\hat{\tilde{O}}$ is constructed as the $N=1$ superspace generalization of the bosonic operator $\tilde{O}$ in eq.(2.29). The anomalous conformal dimension of the super-operator $\hat{\tilde{O}}$ coincides with $\hat{\Delta}_{0}$ in eq.(3.3). In order to have the same $k$-dependence in the super-Virasoro central charge, we can tensor the given two super-cosets with one super-WZNW model on $S U(2)$ at level $l=-k$. This means that $k$ must be negative integer. This compact space will be a part of the five-dimensional compact space $\Sigma^{5}$ formed by $y^{n}$. Since $S U(2)$ is a three-dimensional space, the $\Sigma^{5}$-compact space has to have the following structure

$$
\Sigma^{5}=S U(2) \times \Sigma^{2}
$$

where $\Sigma^{2}$ is a two-dimensional compact space with $\hat{c}_{\Sigma^{2}}=2$. The compact space $\Sigma^{2}$ can be realized either as a torus or a sphere. So the target space of the given string model at the UV-point will be $M^{D} \times S U(2) \times \Sigma^{2} \times$ Super-Liouville.

There is another interesting solution for $\Sigma^{5}$. Instead of $S U(2) \times \Sigma^{2}$, we can take the following coset

$$
T^{1,1}=\frac{S U(2) \times S U(2)}{U(1)}
$$

If we take the Kac-Moody level of each of these two $S U(2)$ s being equal to $-2 k$, then we get the right $k$-dependence in the Virasoro central charge. It is worth mentioning that this space has the symmetry of the 5-dimensional Einstein space [27] recently discussed in the context of the AdS/CFT-duality [28]. This fact seems to be quite intriguing.

The similar procedure allows us to construct zigzag-invariant strings in $D=5, D=6$ and $D=7$ dimensions. Also it is quite curious that the mentioned above tensoring does not permit us to have $M^{D}$ with $D>7$. The dimension seven emerges as the maximally possible dimension of the $N=1$ zigzag-invariant string ?. Indeed, in order to get $D=8$, we would have to take two $S L(2)$ and one $\frac{S L(2)}{U(1)}$. To get the right dependence on $k$ in the

\footnotetext{
*It might be interesting to see whether this restriction on the dimensionality of $M^{D}$ has anything to
} do with the restriction on the dimensionality of simple super-anti-De Sitter supergroups, $d \leq 7$ [29]. 


\begin{tabular}{|c|c|c|}
\hline$D$ & $M^{D}$ & $\Sigma^{9-D}$ \\
\hline 2 & $S L(2) / U(1)$ & $\Sigma^{7}$ \\
\hline 3 & $S L(2)$ & $\Sigma^{6}$ \\
\hline 4 & $\frac{S L(2)}{U(1)} \times \frac{S L(2)}{U(1)}$ & $S U(2) \times \Sigma^{2}, T^{1,1}$ \\
\hline 5 & $S L(2) \times \frac{S L(2)}{U(1)}$ & $S U(2) \times \Sigma^{1},\left[\frac{S U(2)}{U(1)}\right]^{2}$ \\
\hline 6 & $S L(2) \times S L(2),\left[\frac{S L(2)}{U(1)}\right]^{3}$ & $S U(2)$ \\
\hline 7 & $S L(2) \times\left[\frac{S L(2)}{U(1)}\right]^{2}$ & $\frac{S U(2)}{U(1)}$ \\
\hline
\end{tabular}

Table 1: $M^{D} \times \Sigma^{9-D} \times($ Super)Liouville-geometry of the target space before deformation. Here $M^{D}$ is the space which is deformed by the operator $\hat{O}$, one for each factor in case when $M^{D}$ is a product, $\Sigma^{9-D}$ is the compact space formed by $y^{m}$.

super-Virasoro central charge, we then would need at least one $S U(2)$ in the $y^{n}$-sector. However, the latter has to have $\hat{c}_{y} \leq 1$, which would be impossible to get.

All in all we can draw Table 1 for $N=1$ supersymmetric CFTs which lead us to the $N=1$ zigzag-invariant strings in $D$ dimensions.

It is interesting to point out that all $M^{D} \times \Sigma^{9-D} \times$ Super-Liouville spaces are tendimensional. In other words, the deformed super-CFT coupled to $N=1$ supergravity describes certain ten-dimensional non-trivial backgrounds of the critical superstring. All these backgrounds have to be solutions of ten-dimensional supergravity compactified on various spaces. Therefore, we may expect that this theory is unitary, despite the fact that we consider non-unitary WZNW models. The signature of $M^{2}, M^{4}, M^{5}, M^{6}$ and $M^{7}$ depends on whether we gauge away the compact or non-compact $U(1)$ subgroup of $S L(2)$.

\section{Conclusion}

By using the method of perturbed CFT, we have managed to give the world-sheet formulation of the zigzag-invariant string in various dimensions. Within our approach, the zigzag symmetry imposes very strict conditions on the geometry and dimensionality of the 
target space. We considered here only one charge deformation - it will be interesting to study the case when model is deformed by several relevant operators. We did not discuss here why the $S L(2)$ group manifold plays such a special role and is there any connection between this $\operatorname{SL}(2)$ and hidden $\operatorname{SL}(2)$ in the Liouville theory, is there an enhancement of the target space symmetry at the horizon, what are the correlation functions of deformed model, as well as many other important questions which we are planning to discuss in further publications. We do not claim also that our approach based on a gravitational dressing of perturbed world-sheet CFT covers all possible string solutions consistent with the zigzag symmetry. It might be the case that there are some more non-trivial zigzaginvariant string backgrounds (maybe with $D>7$ ) which can not be studied within perturbation theory. Nevertheless we think that this approach can be useful for understanding the relation between gauge field theories and string theory.

\section{Acknowledgments}

I.I.K. thanks A.M. Polyakov for stimulating discussion about confining strings. O.S. would like to thank PPARC for financial support.

\section{Appendix}

In this appendix we would like to discuss a possibility of constructing zigzag-invariant strings based on deformations of a generic coset construction $G / H$. The procedure consists of two steps. The first one is to take into account the gauge dressing of the kinetic term of the WZNW model on $G$ with respect to the gauge group $H$ [30]. The second step is to take into account the gravitational dressing of the deformed gauged WZNW model.

Within the $1 / k$-method, we find the gauge-dressed anomalous conformal dimension of the kinetic term

$$
\Delta_{g . d .}=1-\frac{c_{V}(G)-c_{V}(H)}{|k|}+\ldots
$$

Correspondingly, the Virasoro central charge of the $G / H$ coset is given as follows

$$
c_{G / H}=\operatorname{dim} G-\operatorname{dim} H+\frac{c_{V}(G) \operatorname{dim} G}{|k|}-\frac{c_{V}(H) \operatorname{dim} H}{|k|}+\ldots
$$


Taking into account the gravitational dressing gives rise to the following zigzaganomaly cancellation condition

$$
c_{V}(G)(\operatorname{dim} G-3)=c_{V}(H)(\operatorname{dim} H-3) .
$$

In case of ordinary Lie algebras, this equation has only one solution. Namely,

$$
\operatorname{dim} G=3 \quad c_{V}(H)=0,
$$

which is nothing but $S L(2) / U(1)$ which we have already discussed in the paper. However, in case of super-algebras [31], there exist many other solutions of the type $G / S L(2)$, when $G$ is a supergroup with $c_{V}(G)=0$ and $S L(2)$ is an ordinary Lie group. It migh be interesting to look for solutions similar to the supergeometry considered in [32].

\section{References}

[1] A. M. Polyakov, String theory and quark confinement, hep-th/9711002.

[2] E. Álvarez, C. Gómez and T. Ortín, String representation of Wilson loops, hepth/9806075.

[3] J. Maldacena, The large N-limit of superconformal field theory and supergravity, hepth/9711200.

[4] S. S. Gubser, I. R. Klebanov and A. M. Polyakov, Gauge theory correlators from non-critical string theory, hep-th/9802109.

[5] E. Witten, Anti De Sitter space and holography, hep-th/9802150.

[6] C. Schmidhuber, Nucl. Phys. B404 (1993) 342, hep-th/9212075.

[7] I. R. Klebanov, I. I. Kogan and A. M. Polyakov, Phys. Rev. Lett. 71 (1993) 3243, hep-th/9309106.

[8] O. A. Soloviev, Phys. Lett. B321 (1994) 365, hep-th/9310197.

[9] V. G. Knizhnik and A. B. Zamolodchikov, Nucl. Phys. B247 (19984) 83. 
[10] O. A. Soloviev, Nucl. Phys. B431 (1994) 206.

[11] A.B. Zamolodchikov,Sov. J. Nucl. Phys. 46 (1987) 1090.

[12] J. L. Cardy, in Phase transition and critical phenomena, V.11, eds. C. Domb and J. L. Lebowitz (Academic Press, 1987).

[13] A. Stern, Int. J. Mod. Phys. A5 (1990) 415.

[14] A. M. Polyakov, Mod. Phys. Lett. A2 (1987) 893.

[15] V. G. Knizhnik, A. M. Polyakov and A. B. Zamolodchikov, Mod. Phys. Lett. A3 (1988) 819 .

[16] F.David, Mod.Phys.Lett. A 3, 1988, 1651.

[17] J. Distler and H. Kawai, Nucl. Phys. B329 (1989) 509;

J. Distler, Z. Hlousek and H. Kawai, Int. J. Mod. Phys. A5 (1990) 1093.

[18] Y.Tanii, S. Kojima and N. Sakai, Phys. Lett. B322 (1994) 59.

[19] J.Ambjorn and K. Ghoroku, Int. J. Mod. Phys. A9 (1994) 5689.

[20] C. Schmidhuber and A. A. Tseytlin, Nucl. Phys. B426 (1994) 187, hep-th/9404180;

C. Schmidhuber, RG flow in $2 d$ field theory coupled to gravity, hep-th/9412051.

[21] H. Dorn, Phys. Lett. B343 (1995) 81, hep-th/9512023.

[22] O. A. Soloviev, Nucl. Phys. B440 (1995) 373.

[23] P. Di Vecchia, V. G. Knizhnik, J. L. Petersen and P. Rossi, Nucl. Phys. B253 (1985) 701 ;

E. Abdalla and M. C. B. Abdalla, Phys. Lett. 152B (1985) 59.

[24] A. M. Polyakov and A. B. Zamolodchikov, Mod. Phys. Lett. A3 (1988) 1213.

[25] J. Distler, Z. Hlousek and H. Kawai, Int. J. Mod. Phys. A5 (1990) 391.

[26] M. T. Grisaru and D. Zanon, Phys. Lett. B353 (1995) 64, hep-th/9502094. 
[27] L. J. Romans, Phys. Lett. B153 (1985) 392.

[28] I. R. Klebanov and E. Witten, Superconformal field theory on threebranes at a CalabiYau singularity, hep-th/9807080;

S. S. Gubser, Einstein manifold and conformal field theories, hep-th/9807164.

[29] M. Gunaydin, Unitary supermultiplets of $O S p(1 / 32)$ and M-theory, hep-th/9803138.

[30] I. I. Kogan, A. Lewis and O. A. Soloviev, Int. J. Mod. Phys. A12 (1997) 2425, hepth/9607048; Nucl. Phys. Proc. Suppl. 56B (1997) 154, hep-th/9611208; Int. J. Mod. Phys. A13 (1998) 1345.

[31] V. G. Kac, Commun. Math. Phys. 53 (1977) 31; V. G. Kac and M. Wakimoto, Integrable highest weight modules over affine superalgebras and number theory, hepth/9407057.

[32] R. R. Metsaev and A. A. Tseytlin, Type IIB superstring action in $A d S_{5} \times S^{5}$ background, hep-th/9805028. 